\title{
Effect of Granules Properties on the In-vitro and In-vivo Performance of Ibuprofen Sustained Release Matrix Tablets
}

\author{
M. S. Shubair
}

Faculty of Pharmacy, University of Petra, P.O. Box: 961343, Amman, Jordan

\begin{abstract}
The impact of variations in the wet granulation step during the manufacturing process on the in-vitro and in-vivo performance of ibuprofen sustained release matrix tablets was investigated. Two batches were produced under different wet granulation conditions. The granules of the first batch (T1) were characterized by having a lower bulk density $(0.56 \mathrm{~g} / \mathrm{ml})$, a higher percentage of fines $(56.7 \% \mathrm{w} / \mathrm{w})$ and a smaller geometric mean diameter (dg), $600 \mu \mathrm{m}$. While the granules of batch (T2) were characterized by having a more coherent properties, a higher bulk density $(0.66 \mathrm{~g} / \mathrm{ml})$, a lower percentage of fines $(36.9 \% \mathrm{w} / \mathrm{w})$ and a larger $\mathrm{dg}, 720$ $\mu \mathrm{m}$. Three large scale production batches (B1, B2, B3) were manufactured similarly to $\mathrm{T} 2$ and found to have granules possessing similar properties. In-vitro tests showed that tablets of $\mathrm{T} 1$ had a statistically significant higher release rate constant than tablets of either T2, B1,B2 or B3. In-vivo tests were done using T1 and T2 tablets. Although T1 and T2 were bioequivalent with respect to Cmax and AUC, T2 exhibited a statistically significant longer sustained release characteristics than T1 $(\mathrm{P}<0.05)$.
\end{abstract}

\section{Keywords}

Ibuprofen, Sustained release tablets, Granulation variables, Matrix tablets, In-vitro and In-vivo studies.

\section{Introduction}

Ibuprofen (IB) is a propionic acid derivative of a non-steroidal antiinflammatory drug (NSAID) widely used for the treatment of rheumatoid arthritis and as an analgesic and antipyretic agent [1]. For a short biological half life NSAIDs, a sustained release dosage forms are desirable in order to allow twice or once daily administration of the drug to reduce side effects due to high plasma 
concentration and to improve patient compliance. Different sustained release dosage forms for ibuprofen were proposed [2-7]. One of the designs, which was successful in the formulation of sustained release IB dosage form, was the bimodal drug release pattern $[8,9]$. The bimodal design aimed at delivering a proportion of IB at colon, and consequently a high morning drug plasma concentration might be achieved [8]. The benefit of a high early morning levels of drug in plasma is to overcome morning stiffness symptom of rheumatoid disease. It was found that the choice of matrix material, amount of drug incorporated in matrix, additives type and amounts, the hardness of the tablet, density variation and tablet shape could affect the release rate and the mechanism of release of the drug [10].

The aim of this study was to investigate the effect of variation in granules properties on the in-vitro and in-vivo performance of IB sustained release matrix tablets.

\section{Results and Discussion}

All tablet products were found complying with the required specifications of assay, weight variation, thickness and hardness. The results of the physical properties of $\mathrm{T} 1, \mathrm{~T} 2, \mathrm{~B} 1, \mathrm{~B} 2$ and B3 tablets are shown in tab.1 which indicated reproducibility of the tabletting process.

\begin{tabular}{lccccc}
\hline & $\mathrm{T} 1$ & $\mathrm{~T} 2$ & $\mathrm{~B} 1$ & $\mathrm{~B} 2$ & $\mathrm{~B} 3$ \\
\hline Granules : & & & & & \\
$\mathrm{dg}(\mu \mathrm{m})$ & 600 & 720 & 710 & 740 & 700 \\
$\sigma \mathrm{g}(\mu \mathrm{m})$ & 20.84 & 21.53 & 21.23 & 20.94 & 17.80 \\
\% fraction $<600 \mu \mathrm{m}$ & 56.7 & 36.9 & 41.0 & 35.8 & 30.0 \\
Bulk density $(\mathrm{g} / \mathrm{ml})$ & 0.56 & 0.66 & 0.66 & 0.71 & 0.64 \\
Tablets : & & & & & \\
Weight $(\mathrm{mg})$ & 1096.0 & 1093.3 & 1097.0 & 1099.0 & 1100.0 \\
& \pm 29.56 & \pm 10.38 & \pm 10.46 & \pm 9.45 & \pm 12.00 \\
Hardness $(\mathrm{Kp})$ & 19.3 & 20.3 & 19.7 & 20.0 & 18.7 \\
& \pm 0.79 & \pm 1.15 & \pm 2.30 & \pm 1.05 & \pm 2.21 \\
Thickness $(\mathrm{mm})$ & 7.32 & 7.30 & 7.33 & 7.28 & 7.34 \\
& \pm 0.096 & \pm 0.051 & \pm 0.034 & \pm 0.043 & \pm 0.049 \\
(mean $+\mathrm{SD}, \mathrm{n}=10)$ & & & & &
\end{tabular}

Tab. 1. Physical properties of granules and tablets. 


\section{Granules properties}

\section{Micoroscopical and Density Properties of granules}

Microscopical examination showed that T1 granules were more irregular in shape than T2 granules. Visual inspection of granules indicated that T2 granules were more coherent than T1 granules and indicated also from the bulk density of 0.56 and $0.66 \mathrm{~g} / \mathrm{ml}$ for $T 1$ and $T 2$ respectively. The bulk density of the granules of B1, B2, or B3 (production batches) was similar to T2 (tab.1). T1 granules exhibited the highest percentage of fines $(<600 \mu \mathrm{m})$. B1, B2 and B3 granules had similar physical properties to $T 2$ which indicated a reproducible granulation process was attained with acceptable batch to batch variation. This was not the case with T1 as the wet granulation process was different which resulted in production of granules possessing different physical properties as shown in tab.1.

\section{Dissolution properties of granules}

The dissolution results of $\mathrm{T} 1$ and $\mathrm{T} 2$ granules showed $\mathrm{T} 1$ releasing the drug faster than T2 due to the presence of higher percentage of fines, where $71.7 \%$ $( \pm 1.96 \%)$ and $55.2 \%( \pm 0.25 \%)$ of IB were released after 15 minutes of dissolution for T1 and T2 respectively. After 60 minutes of dissolution T1 released $94.2 \%$ $( \pm 0.54 \%)$ of IB, while T2 released $86.1 \%( \pm 0.35 \%)$ of IB.

\section{Dissolution of the dosage forms}

In-vitro dissolution profiles of IB from 6 different products are shown in fig.1.

\section{Dissolution Models}

The reference product showed the highest dissolution rate. Tab. 2 shows the mathematical modeling parameters and regression data of the dissolution results. It was noticed that the dissolution data did not fit neither the zero-order nor the firstorder kinetics $\left(r^{2}<0.99\right)$. Furthermore, it was notable that the dissolution profiles fitted the Higuchi model $\left(r^{2}>0.99\right)$ indicating that within the limitation of the model, the dissolution data were consistent with a diffusional mechanism of release. 


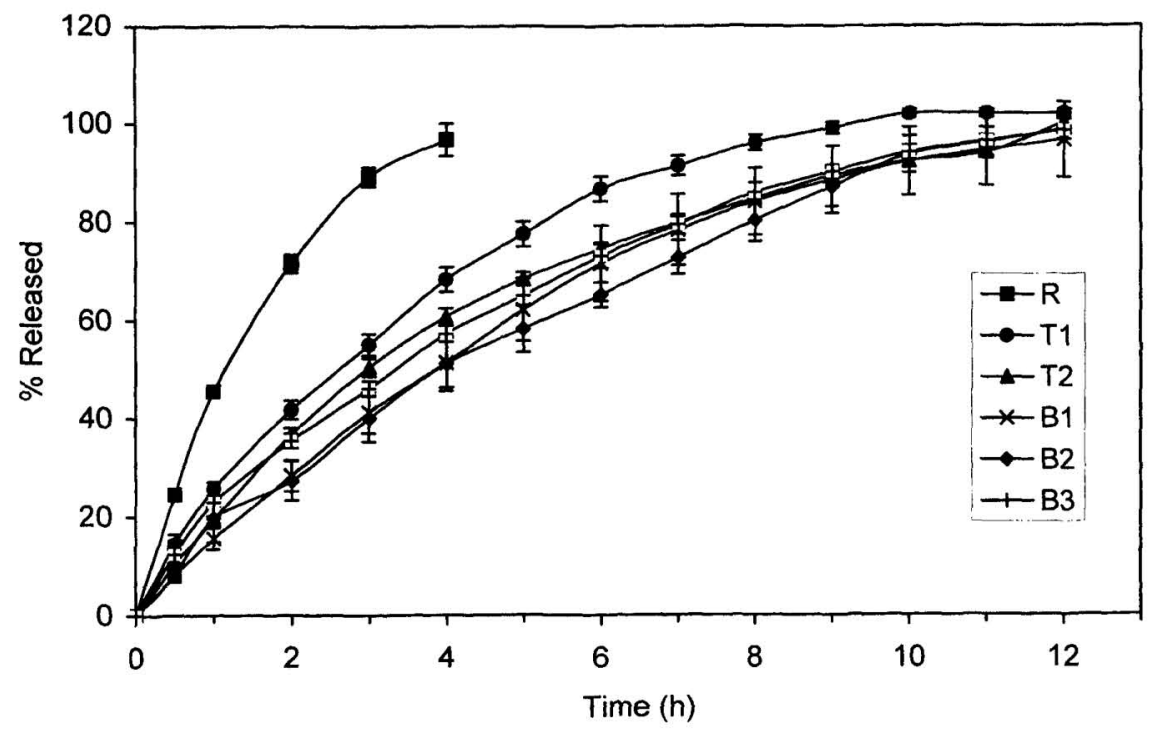

Fig.1. Dissolution of ibuprofen from: Fenbid ${ }^{\oplus}$ capsules (reference, R) and test sustained release tablets ( $T 1$ and $T 2$ as pilot batches; B1, B2 and B3 as production batches), mean $\%$ released $\pm S D, n=6$.

However, matrix dissolution or erosion, which is an important characteristic of swellable and erodible systems is not considered in Higuchi model kinetics. Therefore, additional analysis was done using Korsmeyer-Peppas and HixsonCrowell models to make more definitive conclusions. According to the KorsmeyerPeppas semi-emperical exponential equation, the best overall function was an anomalous non-Fickian transport mechanism $(0.5<n<1.0)$ as shown for all dissolution profiles in tab. 2 which means more than one type of release mechanisms could be involved. Furthermore, it appears that Hixson-Crowell model resulted in a good fit $\left(r^{2}>0.99\right)$. Consequently, erosion of the matrix contributed to the release of the drug along with the diffusional mechanism. 


\begin{tabular}{ccccccc}
\hline Release & $\mathrm{R}$ & $\mathrm{T} 1$ & $\mathrm{~T} 2$ & $\mathrm{~B} 1$ & $\mathrm{~B} 2$ & $\mathrm{~B} 3$ \\
\hline $\begin{array}{c}\text { Zero Order : } \\
\mathrm{r}^{2}\end{array}$ & 0.9378 & 0.9331 & 0.9295 & 0.9465 & 0.9703 & 0.9440 \\
$\mathrm{~K}$ & 0.341 & 0.156 & 0.125 & 0.137 & 0.132 & 0.126 \\
First Order : & & & & & & \\
$\mathrm{r}^{2}$ & 0.8215 & 0.9331 & 0.7631 & 0.7960 & 0.8070 & 0.8014 \\
$\mathrm{~K}$ & 0.0026 & 0.0013 & 0.0012 & 0.0014 & 0.00134 & 0.00114 \\
Hixson-Crowell: & & & & & & \\
$r^{2}$ & 0.9994 & 0.9992 & 0.9963 & 0.9992 & 0.9959 & 0.9980 \\
$\mathrm{~K}$ & 0.0131 & 0.0062 & 0.0044 & 0.0044 & 0.0039 & 0.0046
\end{tabular}

Korsmeyer-

Peppas:

$\begin{array}{lllllll}\mathrm{r}^{2} & 0.9916 & 0.9982 & 0.9924 & 0.9944 & 0.9785 & 0.9954 \\ \mathbf{n} & 0.7726 & 0.7434 & 0.8340 & 0.8741 & 0.8059 & 0.6528\end{array}$

Higuchi Model :

$\begin{array}{ccccccc}r^{2} & 0.9979 & 0.9958 & 0.9901 & 0.9908 & 0.9953 & 0.9938 \\ \mathrm{~K} & 8.150 & 5.146 & 4.296 & 4.446 & 4.367 & 4.199 \\ \text { Intercept } & -19.00 & -10.34 & -10.97 & -17.34 & -16.48 & -9.30 \\ \text { Lag time (min) } & 2.3 & 2.0 & 2.6 & 3.9 & 3.8 & 2.2\end{array}$

$r^{2}$, correlation coefficient; $K$, release rate constant and $n$, diffusional exponent.

Tab. 2. Mathematical model parameters and regression data of the dissolution results.

\section{In-vitro Evaluation}

The similarity factor $\left(f_{2}\right)$ showed significant deviations from the acceptance limits for the comparison of T1 and T2 products with the reference products (R). These results indicated $T 1$ and $T 2$ products were not similar to $R$. The dissolution profiles of $B 1, B 2$ and $B 3$ were found to be similar to that of $T 2 . f_{2}$ values were $65.1,60.3$ and 80.3 for $B 1, B 2$ and $B 3$ respectively which indicated an acceptable batch to batch variation. However, this was not the case with $\mathrm{T} 1$ as the metric value 
was found outside the recommended limits of similarity, $47.1 \%$ (acceptance criteria is $50 \%$ or more).

The difference between $\mathrm{T} 1$ and other test products (T2, B1, B2 and B3) with regard to the time required for $100 \%$ release of IB was found to be significant $(P<0.05)$.

The rate constant of Higuchi's model for T1 was higher than that of eitherT2, B1, B2 or B3 as shown in tab. 2 indicating a faster diffusion rate for T1 tablets.

Furthermore, it was observed visually that upon dissolution $\mathrm{T} 1$ tablets eroded faster than T2. The rate constants of Hixson-Crowell model showed T1 had $K_{\beta}$ value 1.5 times of eitherT2, B1, B2 or B3, an indication of faster erosion. A faster diffusion and erosion of $\mathrm{T} 1$ tablets could be related to the physical properties of their granules as shown previously.

In-vivo study: Bioavailability of $R, T 1$ and $T 2$

Fig. 2 shows the mean $( \pm S D$ ) plasma concentration-time profiles of IB after $600 \mathrm{mg}$ single-dose administration of R, T1 and T2 products.

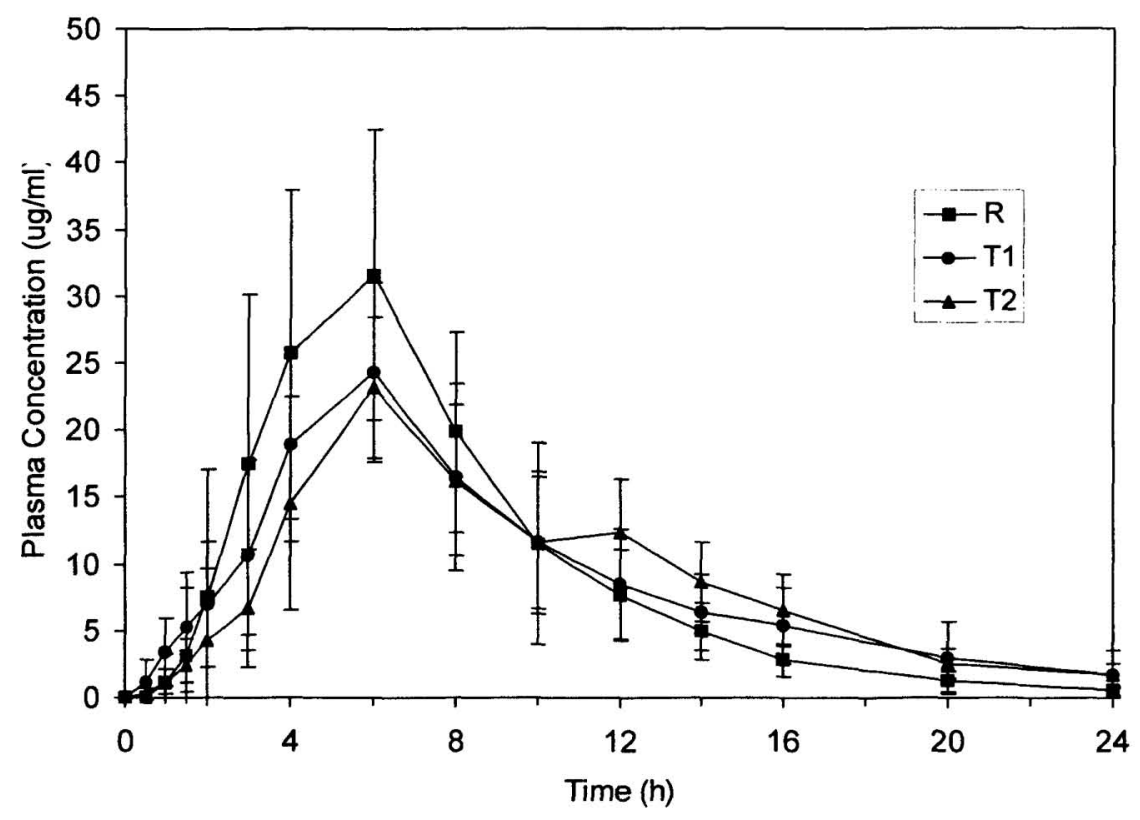

Fig. 2. Bioavailability of ibuprofen from $2 \times 300 \mathrm{mg} \mathrm{Fenbid}^{\oplus}$ capsules (reference, R) and test $600 \mathrm{mg}$ sustained release tablets (T1 and T2). 
Pharmacokinetic parameters derived from the plasma drug data are summarized in tab. 3.

\begin{tabular}{|c|c|c|c|}
\hline Parameter & $\begin{array}{l}\text { R Capsules } \\
2 \times 300 \mathrm{mg}\end{array}$ & $\begin{array}{c}\text { T1 Tablets } \\
600 \mathrm{mg} \\
\end{array}$ & $\begin{array}{c}\text { T2 Tablets } \\
600 \mathrm{mg} \\
\end{array}$ \\
\hline $\mathrm{C}_{\max } \mu \mathrm{g} / \mathrm{ml}$ & $35.1 \pm 10.26$ & $26.1 \pm 6.82$ & $24.2 \pm 5.53$ \\
\hline$t_{\max }(h)$ & $5.7 \pm 1.34$ & $5.6 \pm 1.27$ & $6.2 \pm 1.14$ \\
\hline $\mathrm{AUC}_{0-12} \mu \mathrm{g} \cdot \mathrm{h} / \mathrm{ml}$ & $198.9 \pm 54.58$ & $162 \pm 40.79$ & $147.7 \pm 38.58$ \\
\hline $\mathrm{AUC}_{0-24} \mu \mathrm{g} \cdot \mathrm{h} / \mathrm{ml}$ & $230.1 \pm 62.31$ & $215.1 \pm 58.25$ & $210.6 \pm 53.70$ \\
\hline $\mathrm{AUC}_{0-\infty} \mu \mathrm{g} \cdot \mathrm{h} / \mathrm{ml}$ & $232.4 \pm 62.32$ & $222.0 \pm 61.92$ & $221.7 \pm 62.55$ \\
\hline AUC $_{12-24} \mu \mathrm{g} . \mathrm{h} / \mathrm{ml}$ & $31.5 \pm 14.56$ & $52.9 \pm 25.93$ & $62.9 \pm 21.81$ \\
\hline $\operatorname{Ke}\left(h^{-1}\right)$ & $0.222 \pm 0.900$ & $0.222 \pm 0.044$ & $0.158 \pm 0.060$ \\
\hline$t_{1 / 2}(h)$ & $3.17 \pm 0.136$ & $3.80 \pm 0.403$ & $4.45 \pm 0.191$ \\
\hline MRT $_{\text {in-vivo }}(\mathrm{h})$ & $7.83 \pm 0.350$ & $8.96 \pm 0.520$ & $10.55 \pm 0.380$ \\
\hline$M R T_{\text {in-vitro }}(h)$ & $0.413 \pm 0.0177$ & $0.460 \pm 0.0234$ & $0.558 \pm 0.0293$ \\
\hline $\mathrm{MDT}_{\text {in-vivo }}(\mathrm{h})$ & $5.20 \pm 0.346$ & $6.33 \pm 1.646$ & $7.91 \pm 1.139$ \\
\hline $\mathrm{C}_{12} \mu \mathrm{g} / \mathrm{ml}$ & $7.6 \pm 3.42$ & $8.6 \pm 4.14$ & $12.3 \pm 4.00$ \\
\hline$H V D_{t 50 \%} C_{\max }(h)$ & $5.4 \pm 0.91$ & $6.2 \pm 1.57$ & $7.9 \pm 2.36$ \\
\hline$\% \mathrm{C}_{12} / \mathrm{C}_{\max }$ & $23.2 \pm 11.21$ & $32.9 \pm 14.33$ & $53.1 \pm 19.84$ \\
\hline
\end{tabular}

$A \cup C_{0-12}, A \cup C_{0-24}$ and $A \cup C_{0-\infty}$ : area under the plasma concentration-time curve for 12,24 hours and to the infinity respectively; $C_{\max }$ : mean maximum plasma drug concentration; $\mathrm{C}_{12}$ : mean plasma drug concentration 12 hours after drug administration; $t_{\max }$ : time to maximum concentration; $\mathrm{Ke}$ : elimination rate constant; $t_{1 / 2}$ : elimination half life; $\mathrm{MRT}_{\text {in-vivo }}$ : mean residence time in-vivo; $\mathrm{MRT}_{\text {in-vitro }}$ : mean residence time in-vitro; MDT: the mean dissolution time in-vivo $=\mathrm{MRT}_{\text {in-vivo }}$ (tablets) 2.63 (MRT $_{\text {in-vivo }}$ for ibuprofen solution according to reference 17 ); $H V D_{t 50 \%} C_{\max }$ : half value duration, the time range which $50 \%$ of the observed maximum plasma concentration is attained; $A \mathrm{CC}_{12-24}$ : area under the plasma concentration-time curve from 12-24 hours after drug administration.

Tab. 3. Pharmacokinetic parameters (mean $\pm S D, n=10$ ) obtained from concentrationtime data for reference (R) and two test Products (T1 and T2) after a single dose of $600 \mathrm{mg}$ ibuprofen as a sustained release dosage form. 


\section{Test products versus Reference product}

Peak plasma concentration attained by the products $T 1$ and $T 2$ are significantly lower than the reference $R(P<0.05)$. No significant difference was observed for the values of $t_{\max }$ within the three products $(P>0.05)$. The extent of absorptions $\left(\mathrm{AUC}_{0-24}\right.$ and $\left.\mathrm{AUC}_{0-\infty}, \mu \mathrm{g} . \mathrm{h} . / \mathrm{ml}\right)$ for $\mathrm{R}$ when compared with $\mathrm{T} 1$ and $\mathrm{T} 2$ were found not statistically significantly different $(P>0.05)$. Elimination rate constant $(k e)$ and elimination half life $\left(t_{1 / 2}\right)$ values for $R$ and $T 1$ showed a difference that was not statistically significant. $K e$ and $t_{1 / 2}$ were significantly different for $T 2$ which clearly reflected product-related differences in drug release as shown by its dissimilar in-vivo profiles (fig. 2). The $90 \%$ confidence intervals based on parametric testing of the log-transformed data of the ratio T/R for the $\mathrm{C}_{\max }$ were 0.67-0.84 for $\mathrm{T} 1$ and $\mathbf{0 . 6 0 - 0 . 8 2}$ for $\mathrm{T} 2$. They were outside the generally used acceptance criteria for bioequivalence $(0.70-1.43)$. Furthermore, the corresponding values of the extent of absorption (bioavailability) represented by $\mathrm{AUC}_{0-24}$ were 0.85-1.02 for T1 and 0.83-1.03 for T2 which were within the generally used acceptance criteria for bioequivalence $(0.80-1.25)$. Evaluation of $A \cup C_{0-\infty}$ for $T 1$ and $\mathrm{T} 2$ showed similar results.

\section{Test product $T 1$ versus test product $T 2$}

For Cmax and AUC parameters T1 was found bioequivalent to T2 where the $90 \%$ confidence intervals for the geometric mean ratio (T1/T2) were within the acceptance range of bioequivalence requirements, $C_{\max }(1.01-1.04), A C_{0-24}$ (1.001.07) and $A \mathrm{C}_{0-\infty}(0.99-1.01)$.

\section{In-vivo study: Sustained release characteristics of $R, T 1$ and $T 2$}

In-vitro data showed T2 releasing the drug in a rate significantly slower than $\mathrm{T} 1$ (tab. 2). Despite the slower release rate of T2, the bioavailability data indicated that its extent of absorption represented by $\mathrm{AUC}_{0-24}$ and $\mathrm{AUC}_{0-\infty}$ were not significantly different from those values of $\mathrm{T} 1$ or $\mathrm{R}$ and were within the acceptance range of bioequivalence requirements as described in the previous section. In order to describe the sustained release characteristics of the products $M R T_{\text {in-vitro }}, M R T_{\text {in- }}$ vivo, $\mathrm{MDT}_{\text {in-vivo, }} \mathrm{HVD}_{\mathrm{t50 \%}} \mathrm{C}_{\max }, \mathrm{C}_{12}, \% \mathrm{C}_{12} / \mathrm{C}_{\max }$ and $\mathrm{AUC}_{12-24}$ were determined as shown in tab. 3. It is known that the higher values of these parameters represent greater sustained release performance. The mean dissolution time (MDT in-vivo $_{\text {) }}$ for T2 was greater than $M T_{\text {in-vivo }}$ of either $R$ or $T 1$ and showed a difference that was statistically significant $(P<0.05)$. Furthermore, $M R T_{\text {in-vitro }}$ and $M R T_{\text {in-vivo }}$ values of $T 2$ 
were significantly higher than the corresponding values of either R or T1 products $(\mathrm{P}<0.05) . \mathrm{C}_{12}, \% \mathrm{C}_{12} / \mathrm{C}_{\max }$ and $\mathrm{HVD}_{150 \%} \mathrm{C}_{\max }$ values were significantly higher $(p<0.05)$ for $T 2$ than $R$ or $T 1$. The $C_{12}$ value of $T 1$ was not significantly higher than the $C_{12}$ value of $R(P>0.05)$ although the values of $H V D_{150 \%} C_{\max }$ and $\% C_{12} / C_{\max }$ were marginally significant $(P<0.05)$. The difference in these parameters between $\mathrm{T} 2$ and $\mathrm{R}$ or T1 explained the differences in the residual AUC represented by $A \cup C_{12-24}$. The value of $A \cup C_{12-24}$ in case of $T 2$ was significantly higher than the corresponding value of $R(P<0.05)$ or $T 1(P<0.025)$. The higher values of $C_{12}, \%$ $\mathrm{C}_{12} / \mathrm{C}_{\max }, \mathrm{HVD}_{450 \%} \mathrm{Cmax}_{\text {and }} \mathrm{AUC}_{12-24}$ could be due to the arrival of a portion of the tablet in the colon, where it was then disintegrated and ibuprofen was dissolved and absorbed as previously pointed out [9]. However, this characteristic was lost in the case of T1 tablets. T1 product was matrix tablet prepared from smaller particle size and less coherent granules which produced higher dissolution rate (tab. 2) and smaller $M R T_{\text {in-vitro }}$ whereas $\mathrm{T} 2$ matrix tablets were made from larger particle size and more coherent granules, showed slower dissolution rate (tab. 2) and higher $\mathrm{MRT}_{\text {in-vitro }}$. Thus $\mathrm{T} 1$ tablets were expected to erode or disintegrate faster in the gastrointestinal tract with larger proportion in the small intestinal region and allowing minimum proportion to reach the ascending colon. From the results described above, T2 appeared to have a more prolonged in-vivo delivery of IB than $\mathrm{R}$ and $\mathrm{T} 1$.

\section{In-vivo study: Steady state performance}

The advantage of $\mathrm{T} 2$ over $\mathrm{R}$ was shown by reporting that the morning steady-state mean plasma concentrations after administration of $\mathrm{T} 2$ tablets was significantly higher than that for R capsules, being 18.0 and $10.5 \mu \mathrm{g} / \mathrm{ml}$, respectively. This was interpreted as being a result of higher $\mathrm{C}_{12}$ for $\mathrm{T} 2$ tablets [8]. A high morning drug plasma concentration is considered as an advantage because it is useful to overcome morning stiffness, which is characteristic symptoms of many rheumatic conditions [11]. $R$ and $T 2$ products are administered twice daily, so $C_{12}$ as IB plasma concentration is an important pharmacokinetic parameter to be determined. In this investigation as shown in tab. 3 , the $\mathrm{C}_{12}$ of $\mathrm{T} 2$ was significantly higher $(P<0.05)$ than $C_{12}$ of either $R$ or $T 1$. However, $R$ and $T 1$ products did not show significant difference for their $C_{12}$ values $(P>0.05)$. When $M D T_{\text {in-vivo }}$ or $M R T_{\text {in- }}$ vivo was plotted versus $C_{12}$ values of $R, T 1$ and $T 2$ a linear relationship was obtained $\left(0.90<r^{2}<0.99\right)$. Thus, it could be predicted that $T 1$ would produce $C_{12}$ at steady- 
state conditions significantly lower than $\mathrm{C}_{12}$ of $\mathrm{T} 2$. Consequently, the advantages of lower fluctuation of the steady state concentrations and higher $\mathrm{C}_{12}$ value attained by T2 tablets will not be achieved by the administration of T1 tablets.

In conclusion, variations in the wet granulation process were reflected on the in-vitro and in-vivo sustained release characteristics of ibuprofen sustained release matrix tablets. In-vitro, T1 showed a significant higher diffusion and erosion rates than T2 $(P<0.05)$. Although $T 1$ and $T 2$ were bioequivalent with respect to $C \max , A U C_{0-24}$ and $A \cup C_{0-\infty}, T 2$ exhibited a statistically significant longer sustained release characteristics than $T 1(P<0.05)$ as represented by the parameters $C_{12}, M R T_{\text {in-vivo }}$, $M R T_{\text {in-vitro }}, M D T_{\text {in-vivo }}, H V D_{t 50 \%} C_{\max }, C_{12} / C_{\max }$ and $A U C_{12-24}$.

\section{Experimental \\ Materials}

Ibuprofen; lactose monohydrate; maize starch; magnesium stearate; titanium dioxide; polysorbate 80; and talc were materials of pharmaceutical grade and supplied by the Arab Pharmaceutical Manufacturing Co., Sult, Jordan. Ammonio methacrylate copolymer, Type B, NF (Eudragit RS) granules were supplied by Rohm Pharma Polymers, Degussa, Darmstadt, Germany. Hypromellose 2910 (hydroxypropyl methylcellulose), Methocel E15 Premium- 29\% methoxyl and 8.5\% hydroxypropoxyl content and viscosity grade $15 \mathrm{cP}$ was supplied by Colorcon, Kent, UK. Colloidal silicon dioxide (Aerosil 200, Degussa, Germany) and Sodium starch glycolate (Primojel, Avebe America Inc., NJ, USA) were used. All chemicals and solvents were of analytical grade and supplied by E.Merck, Germany. Distilled water was used to prepare aqueous solutions and granulating agents. Fenbid ${ }^{\circledR} 300$ mg ibuprofen sustained release (SR) spansules in capsules (Smithkline Beecham, UK) were purchased locally and used as reference product (R).

\section{Production of tablets}

$600 \mathrm{mg}$ IB sustained release tablets were produced according to the general formula as reported in tab. 4. Five different batches were prepared, T1 and T2 as pilot batches; and B1, B2 and B3 as large scale production batches. The powder mixture was prepared by mixing together IB, half amount of lactose powder and hypromellose in Gral mixer and granulator (Collette, Belgium) for 10 minutes with the mixer adjusted at low speed and the chopper at high speed. T1 was wet granulated with aqueous dispersion of Eudragit RS ( $20 \% \mathrm{w} / \mathrm{w})$ by adding the 


\begin{tabular}{lc}
\hline Ingredients & mg/tablet \\
\hline Core : & \\
lbuprofen & 600.0 \\
Lactose monohydrate & 111.34 \\
Maize Starch & 33.40 \\
Hypromellose & 167.11 \\
Eudragit RS & 111.34 \\
Sodium Starch glycolate & 22.27 \\
Talc & 11.13 \\
Colloidal silicon dioxide & 2.83 \\
Magnesium Stearate & 5.57 \\
Core Weight & 1065 \\
Film Coating : & \\
Hypromellose & 24.0 \\
Talc & 8.0 \\
Titanium dioxide & 2.8 \\
Polysorbate 80 & 0.2 \\
Distilled water & 300 \\
Total Weight & 1100
\end{tabular}

Tab. 4. Formulation composition.

required amount of the dispersion gradually in five portions to the powder mixture and mixed for 3 minutes after each addition. The speed of either mixer or chopper was adjusted at low speed setting. The other batches T2, B1, B2 and B3 were wet granulated similarly using a more diluted Eudragit RS dispersion (15\% w/w) allowing more water to be incorporated into the wet mass. Addition was performed in 6 portions allowing mixing for 5 minutes after each addition. For all batches wet granules were then dried and milled using standard processes. Particle size analysis was done in duplicate on $100 \mathrm{gm}$ of screened granules through sieves $2000,1250,1000,800,600 \mu \mathrm{m}$ and receiver. Shaking was conducted for 15 minutes. Diluent granules made from lactose powder and starch and granulated with hypromellose $5 \%$ aqueous solution were prepared using the same equipment. 
Final powder mix was then prepared by mixing drug granules with diluent granules, Primojel and talc for 15 minutes. Aerosil was then added and mixing was performed for another 5 minutes. At the end of mixing operation magnesium stearate was added and mixed for 5 minutes. The final powder mix was compressed into tablets (oblong, $19 \mathrm{~mm} \times 9 \mathrm{~mm}$ ) using 22 station rotary tabletting machine (Perfecta -5 , Fette, Germany) under controlled hardness $(20 \mathrm{Kp} \pm 10 \%)$. Film coating was done using Accela cota (Manesty, UK). Physical characteristics like tablet weight, thickness and hardness (Core) were controlled.

\section{Quality control tests}

Assay of IB tablets and capsules was done according to the USP 24 monograph. The dissolution profile of each dosage form was obtained using Erweka dissolution apparatus (Hensenstamm, Germany) with paddles rotating at $75 \mathrm{rpm}$ and $900 \mathrm{ml}$ of USP phosphate buffer solution $\left(\mathrm{pH} \mathrm{7.2)}\right.$ ) heated at $37 \mathrm{C}^{\circ}$. For dissolution of granules, paddles were allowed to rotate at $50 \mathrm{rpm}$ instead of $75 \mathrm{rpm}$ and the dissolution medium was diluted with distilled water (2:1) while the $\mathrm{pH}$ was kept at 7.2. Such conditions allowed $100 \%$ of IB to be released in more than 60 minutes.. The drug concentration was determined spectrophotometrically versus a standard solution (DU-7 Spectrophotometer, Beckman, USA) at $275 \mathrm{~nm}$.

\section{In-vivo tests}

Randomized, single dose crossover studies were performed on 10 healthy male volunteers aged between 18 and 40 years over 3 treatment periods with one week washout phase after each period according to a previously published method and followed ICH guidelines [8]. The dose of $600 \mathrm{mg}$ IB (one tablet of either T1 or T2, 2 capsules of Fenbid ${ }^{\circledR}$ as a reference) was given orally with $250 \mathrm{ml}$ of orange juice after having a light breakfast. Withdrawal of blood samples $(7 \mathrm{ml})$ via a cannula inserted into a forearm vein was done immediately before the dose $(0$ time) and then at $0.5,1,1.5,2,3,4,6,8,10,12,14,16,20$ and 24 hours post administration. Blood samples were drawn into heparinised tubes, centrifuged at $3000 \mathrm{rpm}$ for 5 minutes, and the plasma was stored frozen at $-20^{\circ} \mathrm{C}$ until the day of analysis. Plasma analysis for IB concentrations was carried out using a validated HPLC method [8].

\section{In-vitro data analysis}

Drug dissolution from solid dosage forms has been described by different kinetic models like zero order, first order, Higuchi square root of time model, 
Korsmeyer-Peppas semi-empirical exponential equation model and HixsonCrowell cubic root of the unreleased fraction of drug versus time model $[12,13]$.

\section{Evaluation of similarity}

Comparison of dissolution profiles were done using the similarity factor $\left(f_{2}\right)$ as adopted by FDA and EMEA (European Agency for the Evaluation of Medicinal Products) as a criterion for the assessment of the similarity between two in-vitro dissolution profiles $[14,15]$. FDA and EMEA suggest that two dissolution profiles are considered similar if $t_{2}$ value is between 50 and 100 . The test is sensitive to measurements obtained after either test or reference batch are dissolved more than $85 \%$. Shah et al [14] recommended that, the number of sample points be limited not more than one, once any of the product reaches $85 \%$ dissolution.

\section{In-vivo data analysis}

The bioavailability parameters of the three products were determined by a standard non-compartmental method and ANALYSIS OF VARIANCE (ANOVA) statistics were used for bioequivalence evaluations. Pharmacokinetic parameters were calculated. The maximum IB plasma concentration $\left(C_{\max }, \mu \mathrm{g} / \mathrm{ml}\right)$ and the corresponding peak time $\left(t_{\max }, h\right)$ were determined by the examination of the individual drug plasma concentration-time profiles. The area under the curve to the last measurable concentration $\left(\mathrm{AUC}_{0-24}, \mu \mathrm{g} . \mathrm{h} / \mathrm{ml}\right.$ ) and the area under the curve from 0 to 12 hours $\left(A \cup C_{0-12}, \mu \mathrm{g} . \mathrm{h} / \mathrm{ml}\right)$ or from 12 to 24 hours after administration $\left(A \cup C_{12-}\right.$ $24, \mu \mathrm{g} . \mathrm{h} / \mathrm{ml})$ were calculated by the linear trapezoidal rule. $A \cup C_{0-\infty}(\mu \mathrm{g} . \mathrm{h} / \mathrm{ml})$ was calculated as: $\left[\left(A C_{0-24}\right)+\left(C_{t} / K e\right)\right]$ where $C_{t}$ is the last detectable plasma concentration and $\mathrm{Ke}$, is the elimination rate constant $\left(\mathrm{h}^{-1}\right)$. Half Value Duration $\left(\mathrm{HVDt}_{50 \%} \mathrm{C}_{\max }, \mathrm{h}\right.$ ) analysis was used to evaluate the sustained release nature of the product [5]. It is the time range which $50 \%$ of the observed maximum plasma level concentration is attained. $\% \mathrm{C}_{12} / \mathrm{C}_{\max }$, the percentage of the ratio of $\mathrm{C}_{12}$ (the plasma concentration at the end of the intended dose interval) and $C_{\max }$. This ratio provides an indicator of the peak-trough fluctuation to be expected after steadystate administration. A higher percentage also indicates a better performance as a sustained release dosage forms that are given twice daily. In-vivo mean residence time $\left(M R T_{\text {in-vivo }}, h\right)$, in-vitro mean residence time $\left(M R T_{\text {in-vitro }}, h\right)$ and in-vivo mean dissolution time of the product (MDT in-vivo) were calculated according to Banaker [16] and Shargel and Yu [17]. The pharmacokinetic parameters $A \cup C$ and $C_{\max }$ were assumed to be log-normally distributed. Log-transformed values of these 
pharmacokinetic parameters were analyzed by performing ANOVA analyses using SAS statistical program. A $5 \%$ level of significance was used for all comparisons. The two one-sided tests for bioequivalence and $90 \%$ confidence intervals for the ratios of the geometric means were calculated. The recommended range of bioequivalence was $80-125 \%$ for $A U C$ and $70-143 \%$ for $C_{\max }$.

\section{Acknowledgement}

I would like to express my gratitude to Dr. T. Arafat (University of Petra), Dr. N.Idkaidek (JUST), Dr. A.Sallam (APM.Co.) for their cooperation and useful discussion and also to Miss Muna Al-Resheq for her excellent secretarial work in preparing this manuscript.

\section{References}

[1] Reynolds J E F, editor. Ibuprofen.

In: Martindale, the extra pharmacopoeia. $31^{\text {st }} \mathrm{ed}$.

London: The pharmaceutical press. 1996:50.

[2] Kawashima Y, Iwamoto T, Niwa T, Takeuchi H, Hino T. Uniform and improved bioavailability of newly developed and sustained release of ibuprofen microcapsules. Int. J. Pharm.1993;89:9-17.

[3] Zhou F, Vervaet C, Schelkens M, Lefebvre R, Remon J P. Bioavailability of ibuprofen from matrix pellets based on the combination of waxes and starch derivatives. Int. J. Pharm. 1998;168:79-84.

[4] Khan G M, Zhu J B. Studies on drug release kinetics from ibuprofen-carbomer hydrophilic matrix tablets: influence of co-excipients on release rate of the drug.

J. Control. Rel. 1999;57:197-203.

[5] Khan G M, Zhu J B. Ibuprofen release kinetics from controlled release tablets granulated with aqueous polymeric dispersion of ethylcellulose: influence of several parameters and co-excipients. J. Control. Rel. 1998;56:127-34.

[6] Brabandera C, Vervaet C, Grtz J P, Remon J P. Bioavailability of ibuprofen from matrix mini-tablets based on a mixture of starch and microcrystalline wax. Int. J. Pharm. 2000;208:81-6. 
[7] Perumal D.

Microencapsulation of ibuprofen and Eudragit RS- 100 by the emulsion solvent diffusion technique.

Int. J. Pharm. 2001;218:1-11.

[8] Gharaibeh M, Zmeili S, Saket M, Arafat T, Saleh M, Sallam E, Shubair M, Deleq S.

Ibuprofen sustained release dosage forms: Single and multiple dose studies of tablets and spansules in normal male volunteers.

Clin. Drug. Invest. 1996;11:174-83.

[9] Wilson C G, Washington N, Greaves J L, Kamali F, Rees J A, Sempik A k, Lampard J F.

Bimodal release of ibuprofen in a sustained release formulation: a scintigraphic and pharmacokinetic open study in healthy volunteers under different condition of food intake.

Int. J. Pharm. 1989;50:155-61.

[10] Capan Y.

Influence of technological factors on formulation of sustained release tablets. Drug. Dev. Ind. Pharm. 1989;15(6\&7):927-56.

[11] Laska E M, Sunshine A, Marrero I, Olson N, Siegel C, McCormick N. The correlation between blood levels of ibuprofen and clinical analgesic response. Clin. Pharmacol. Ther. 1986;40:1-7.

[12] Costa P, Lobo J M S. Modeling and comparison of dissolution profiles.

Eur. J. Pharm. Sci. 2001;13:123-33.

[13] Siepmann J, Peppas N A. Modeling of drug release from delivery system based on hydroxypropylmethylcellulose (HPMC). Adv. Drug. Deliv. Rev. 2001;48:139-57.

[14] Shah V P, Tsong Y, Sathe P, Liu J. In-vitro dissolution profile comparison: statistics and analysis of the similarity factor $f 2$.

[15] FDA. Pharm. Res. 1998;15(6):889-96.

Extended release oral dosage forms: Development, evaluation and application of in-vitro/in-vivo correlation. In: Guidance for industry. September 1997.

[16] Banakar U V. Pharmaceutical dissolution testing. In: Drug and the pharmaceutical sciences. Volume 49. New York: Mercel Dekker Inc, 1992:375-80. 
332 M. S. Shubair: Effect of Granules Properties on the In-vitro and In-vivo ...

[17] Shargel L, Yu A B C.

Applied biopharmaceutics and pharmacokinetics. $3^{\text {rd }}$ ed.

New Jersey: Prentice- Hall International Inc,1993:505,525.

Received July $1^{\text {st }}, 2004$

Accepted December 3rd, 2004 